\title{
Experiment, Optimization, and Design of Electromagnetic Track Brake for High-Speed Railways System
}

\author{
Chun Xiang, ${ }^{1}$ Jun-Cheng Wang, ${ }^{2}$ Yu-Feng Gu, ${ }^{2}$ Shi-Jin Zhang, ${ }^{2}$ and Shi-An Chen $\mathbb{D}^{1,2}$ \\ ${ }^{1}$ College of Mechanical and Automotive Engineering, Zhejiang University of Water Resources and Electric Power, Hangzhou, \\ China \\ ${ }^{2}$ School of Automobile and Traffic Engineering, Jiangsu University, Zhenjiang, China \\ Correspondence should be addressed to Shi-An Chen; chensa@zjweu.edu.cn
}

Received 23 October 2019; Revised 27 January 2020; Accepted 6 February 2020; Published 9 March 2020

Academic Editor: Alessandro Lo Schiavo

Copyright (c) 2020 Chun Xiang et al. This is an open access article distributed under the Creative Commons Attribution License, which permits unrestricted use, distribution, and reproduction in any medium, provided the original work is properly cited.

To enhance braking force and control convenience of high-speed railway systems, this paper proposes a new electromagnetic track brake, and the corresponding design, optimization, and experimental test are implemented. The proposed track brake is longitudinal-axis magnetic circuits excited by multiple coils electromagnets, and the pole shoes are extending outward. A preliminary design of an electromagnetic track brake is developed, including iron core height, iron core width, iron core gap, excitation ampere-turn, coil arrangement form, coil thickness, and preliminary height of single-layer coil. The electromagnet number and pole shoe gap are optimized through three-dimensional electromagnetic simulation comparisons. The final design of the electromagnetic track brake is determined, including iron core length, copper wire diameter, coil turn, and final height of singlelayer coil. Experimental verification of electromagnetic attractive force is performed through prototype tests, and the newly developed electromagnetic track brake can enhance electromagnetic braking deceleration by $39 \%$.

\section{Introduction}

Since the high-speed rail system Shinkansen was first launched in 1964, such rail systems have developed rapidly in Japan, Germany, France, and China $[1,2]$. The current maximum speed of high-speed trains reportedly reached $574.8 \mathrm{~km} / \mathrm{h} \mathrm{[3].}$ Reducing speed of trains as quickly as possible during emergencies is important. Magnet track brake is employed together with the main wheel-rail brake system in emergency circumstances because it is independent of wheel-rail adhesion $[2,4]$. Moreover, magnet track brake was reported to be used against slippery tracks caused by contaminants such as snow, sand, and leaves, among others [5].

During operation of magnet track brake, its brake shoes are driven by magnets to attract and contact track. A sliding motion occurs between pole shoes and track to generate braking force (BF) [6]. Magnet track brakes can be categorized into permanent-magnet track brake and electromagnetic track brake (ETB), classified by the excitation mode [7]. Magnet circuit of ETB has two layout forms, namely, lateral-axis form and longitudinal-axis form [8].
To enlarge braking force and control convenience of high-speed railway systems, this research present a new ETB. The proposed ETB has two outstanding characteristics. The first characteristic is its use of a longitudinal-axis magnetic circuit excited by electromagnets with multiple coils in parallel. This coil arrangement can achieve step control on $\mathrm{BF}$ to achieve good control convenience when the exciting voltage is fixed. The second characteristic is pole shoes extending out. The extended pole shoes can enlarge the contact area between pole shoes and track to enhance BF. Furthermore, the enlarged contact area can increase brake service life. Safety is the most important factor when an emergency occurs. Therefore, a battery is chosen as an excited power source to ensure working reliability of ETB. As the battery voltage in high-speed railway carriages in China (CRH) is $24 \mathrm{~V}$ [3], a new ETB design, its optimization, and experimental verification are necessary.

The measures of iron core, coil, protection block, and longitudinal beam are ascertained in this research. Iron core gap, coil arrangement form, and excitation ampere-turn are also determined. Electromagnet number and pole shoe gap 
are optimized through simulations to attain the largest electromagnetic attractive force (EAF) $[5,6]$. Finally, an experimental verification of an EAF between the proposed ETB and track is conducted through prototype tests.

The contributions of this study are summarized as follows: (1) a new ETB excited by a battery with longitudinalaxis magnetic circuits and extended pole shoes is proposed; (2) the electromagnet number is optimized to be six, and the pole shoe gap is optimized to be $40 \mathrm{~mm}$, which significantly improve BF; (3) the proposed ETB can enhance braking deceleration by $39 \%$ unlike the one for ICE, thereby improving braking security significantly; and (4) the availability of ETB is experimentally verified, which provides reference for ETB design.

This paper is organized as follows: first, a preliminary design of ETB is developed, including iron core height, iron core width, iron core gap, excitation ampere-turn, coil arrangement form, coil thickness, and the height of the singlelayer coil. Then, simulations are carried out, and optimization results are presented. Second, the final design of the ETB based on the optimization is created, including iron core length, copper wire diameter, coil turns, and the final height of the single-layer coil. Third, the experimental verification of EAF is conducted. Finally, the BF is calculated.

\section{Preliminary Design of ETB}

The iron core and pole shoe widths are decided according to track width. The numerical range of the iron core length is calculated through magnetic circuit analysis. The excitation ampere-turn is obtained according to magnetic circuit analysis, and equivalent air gap between pole shoes and track belong to the preliminary design. The coil arrangement form, winding thickness, coil height, iron core height, pole shoe height, the measures of protection block and longitudinal beam, adjacent iron cores gap, and the gap between protection block and adjacent iron core are determined in this part.

The material of the longitudinal beam, iron core, pole shoe, and protection block is \#10 steel. The materials of the coil and track are copper and \#70 Mn steel, respectively [9].

The directions along the length and width of ETB are in accordance with the longitudinal and lateral direction of track, respectively. The longitudinal length and height of the magnet track brake are limited to the bogie size because ETB is installed under the train bogie. Considering the bogie wheel base and the wheel diameter of electric motor train unit, which are 2500 and $860 \mathrm{~mm}$ [9], respectively, the longitudinal length of ETB is determined as $1400 \mathrm{~mm}$, which is the basic design condition for the following designs.

2.1. Iron Core Parameters. Considering that the largest effective width of the track is $73 \mathrm{~mm}$ [10], the width of the iron core and pole shoe $f$ is ascertained as $75 \mathrm{~mm}$ to achieve the largest working area through electromagnetic field between pole shoes and track.
As exhibited in Figure 1, the magnetic circuit system and magnetic flux leakage are analyzed when the number of electromagnets is assumed to be six.

According to the magnetic circuit principle, the relationship between $\phi_{a}$ and $\phi_{b}$ is expressed as

$$
\phi_{a}=B_{a} S_{a} \leq 2 \phi_{b}=2 B_{b} S_{b},
$$

where $\phi_{a}$ is the magnetic flow through the iron core, $\mathrm{Wb} ; \phi_{b}$ is the magnetic flow through track, $\mathrm{Wb} ; B_{a}$ is the saturation flux density of the electromagnet in point $a, T ; B_{b}$ is the saturation flux density of track in point $b, T ; S_{a}$ is the electromagnet cross-sectional area, $\mathrm{mm}^{2}$; and $S_{b}$ is the rail cross-sectional area, which equals $7800 \mathrm{~mm}^{2}$ [10]. According to B-H curves of \#10 steel and \#70 Mn steel in Figure 2 [11], $B_{a}$ is $1.35 \mathrm{~T}$, and $B_{b}$ is $1.15 \mathrm{~T}$. The iron core length $D_{1}$ satisfies the following constraint:

$$
D_{1} \leq \frac{2 B_{b} S_{b}}{75 B_{a}}=177.2 \mathrm{~mm} .
$$

The final iron core length will be determined later through electromagnet optimization, and it must satisfy the abovementioned constraint equation.

2.2. Calculation of Equivalent Air Gap. Equivalent air gap between pole shoe and track is decided after the excitation ampere-turn of coil is designed. Two kinds of air gap present during the operation of the ETB: the air gap between longitudinal beam and iron cores, which is due to the manufacturing process, and the air gap between pole shoes and track, which is caused by track irregularity, real-time contact status, and track contaminants, among others. For a brief calculation, the following assumptions are made.

(1) The equivalent air gap equals the sum of the abovementioned air gaps, and it is located between pole shoes and track

(2) The distance from the lowermost portion of pole shoes to the uppermost portion of track equals $1 \mathrm{~mm}$ [9]

As shown in Figure 3, the top curve of the lateral cross section of a track is a combinational arc. The half curve mainly consists of Arcs AB, BC, and CD whose radius is 300 , 80 , and $13 \mathrm{~mm}$, respectively [10]. If the coordinate origin is chosen as represented in Figure 3, the coordinates of points $A, B, C$, and $D$ and the three centers of Arcs $\mathrm{AB}, \mathrm{BC}$, and $\mathrm{CD}$ can be determined as listed in Table 1 . Here, $\delta_{\mathrm{ab}}$ is the distance between the pole shoe bottom and track top, which equals $1 \mathrm{~mm}$.

The magnetic induction is a variable due to the alterable gap between pole shoe and track. The air gap between pole shoe and track can be divided with a great number of magnetic tubes. The magnetic induction in these tubes can be considered equivalent when the magnetic tubes are sufficiently small. The magnetic permeability in one tube is expressed as 


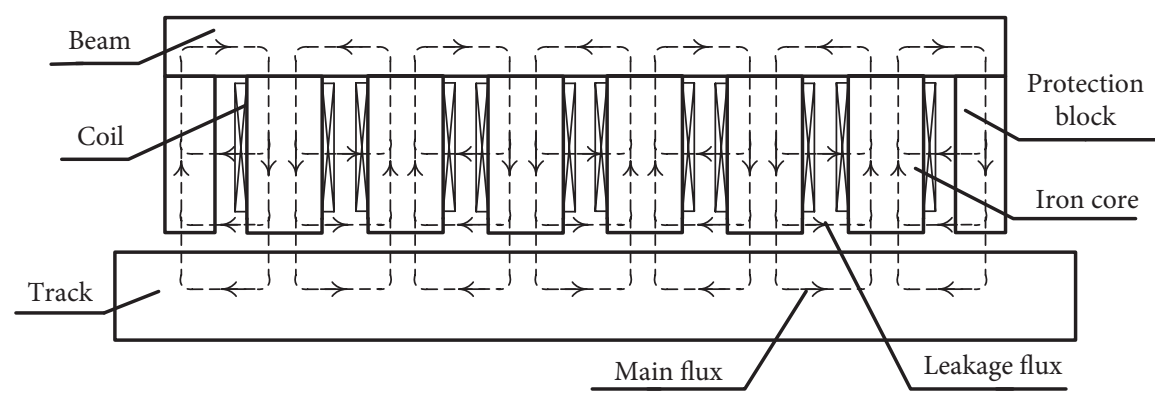

FIgURE 1: Magnetic circuit system of the ETB.

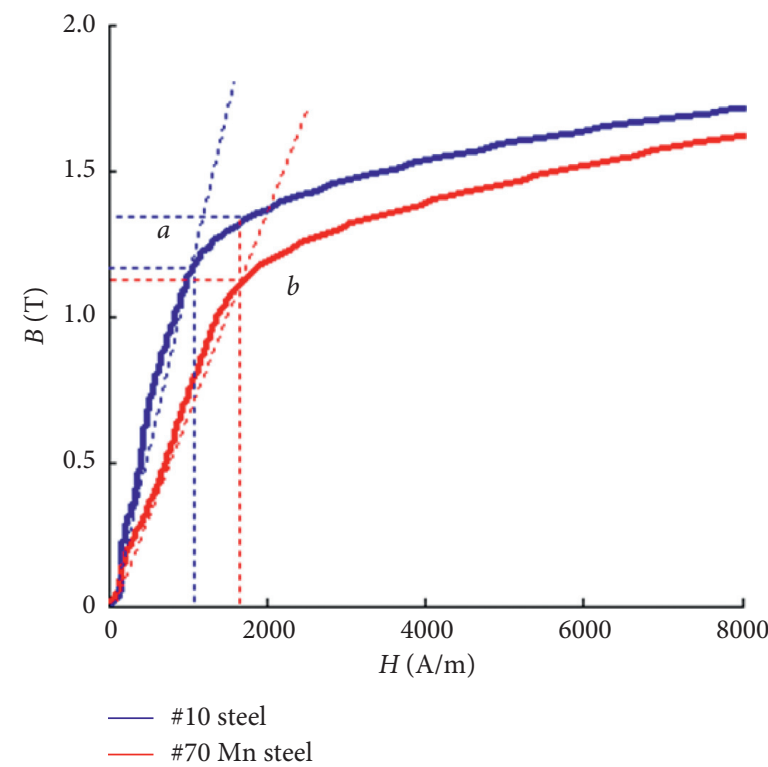

Figure 2: B-H curves of \#10 steel and \#70Mn steel.

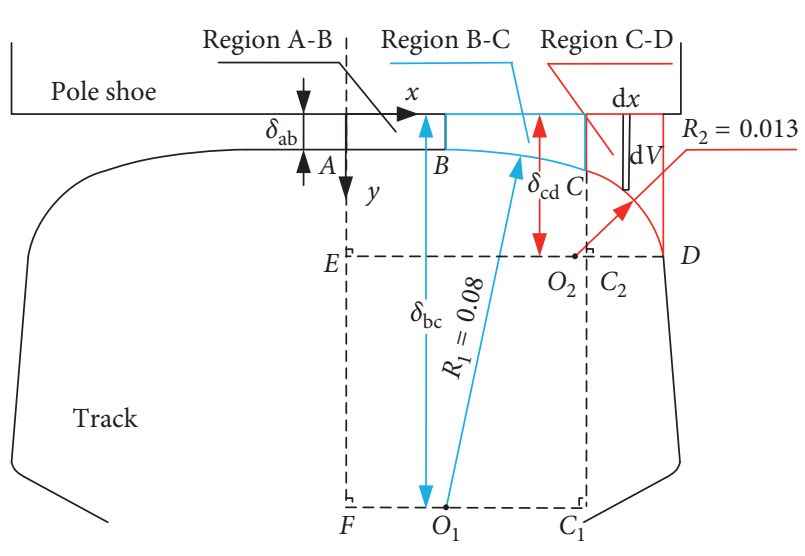

FIGURE 3: Detailed flux tubes pattern between pole shoe and track.

$$
\mathrm{d} G=\mu_{0} \iiint_{V} \frac{\mathrm{d} V}{L_{p}^{2}},
$$

where $L_{p}$ is the tube length, $\mathrm{m}$, and $\mathrm{d} V$ is the tube volume, $\mathrm{m}^{3}$. $G_{\mathrm{AB}}, G_{\mathrm{BC}}$, and $G_{\mathrm{CD}}$ are the magnetic permeability in regions $A$ $B, B-C$, and $C-D$, respectively, and they can be written as follows:

$$
\left.\begin{array}{l}
G_{\mathrm{AB}}=\mu_{0} b \int_{X_{A}}^{X_{B}}\left(Y_{\mathrm{AB}}-\sqrt{R_{1}^{2}-\left(x-X_{\mathrm{AB}}\right)^{2}}\right)^{-1} \mathrm{~d} x \\
G_{\mathrm{BC}}=\mu_{0} b \int_{X_{B}}^{X_{C}}\left(Y_{\mathrm{BC}}-\sqrt{R_{2}^{2}-\left(x-X_{\mathrm{BC}}\right)^{2}}\right)^{-1} \mathrm{~d} x \\
G_{\mathrm{CD}}=\mu_{0} b \int_{X_{\mathrm{C}}}^{X_{D}}\left(Y_{\mathrm{CD}}-\sqrt{R_{3}^{2}-\left(x-X_{\mathrm{CD}}\right)^{2}}\right)^{-1} \mathrm{~d} x
\end{array}\right\},
$$

where $X_{A}, X_{B}, X_{C}$ and $X_{D}$ are the $\mathrm{x}$-coordinates of Points $A$, $B, C$, and $D$; and $\left(X_{\mathrm{AB}}, Y_{\mathrm{AB}}\right),\left(X_{\mathrm{BC}}, Y_{\mathrm{BC}}\right)$ and $\left(X_{\mathrm{CD}}, Y_{\mathrm{CD}}\right)$ are the center coordinates of Arcs AB, BC, and $C D$, respectively. The equivalent air gap between pole shoes and track can be written as

$$
\left.\begin{array}{l}
l_{\mathrm{mg}} \approx \mu_{0} \frac{S_{c}}{2\left(G_{\mathrm{AB}}+G_{\mathrm{BC}}+G_{\mathrm{CD}}\right)} \\
l_{\mathrm{pg}} \approx l_{\mathrm{mg}}
\end{array}\right\},
$$

where $l_{\mathrm{mg}}\left(l_{\mathrm{pg}}\right)$ is the equivalent air gap between pole shoes and track. According to Table 1 and equations (3)-(5), the calculation result of $l_{\mathrm{mg}}\left(l_{\mathrm{pg}}\right)$ is $1.9 \mathrm{~mm}$. 
TABle 1: Coordinates needed in calculating equivalent air gap.

\begin{tabular}{lccccccc}
\hline & $A$ & $B$ & $C$ & $D$ & Center of arc AB & Center of arc BC & Center of arc CD \\
\hline$X(\mathrm{~mm})$ & 0 & 10 & 25.35 & 35.4 & 0 & 7.387 & 22.417 \\
$Y(\mathrm{~mm})$ & $\delta_{\mathrm{ab}}$ & $\delta_{\mathrm{ab}}+0.2$ & $\delta_{\mathrm{ab}}+2.2$ & $\delta_{\mathrm{ab}}+14.2$ & $\delta_{\mathrm{ab}}+300$ & $\delta_{\mathrm{ab}}+80.157$ & $\delta_{\mathrm{ab}}+14.865$ \\
\hline
\end{tabular}

2.3. Preliminary Design of Magnetic Circuit Coil. The magnetic circuits are excited longitudinally by electromagnets. The network topology scheme of magnetic circuits is depicted in Figure 4.

when the electromagnet number equals $n$, there are $(n+1)$ branch magnetic circuits of the longitudinal beam $b_{\mathrm{ak}}$ $(1 \leq \kappa \leq n+1), n$ branch magnetic circuits of the iron cores $b_{\mathrm{ck}}(1 \leq \kappa \leq n)$, two branch magnetic circuits of the protection blocks $b_{\mathrm{pk}}(1 \leq \kappa \leq 2), n$ branch magnetic circuits of the excitation coils $b_{\mathrm{ek}}(1 \leq \kappa \leq n), n$ branch magnetic circuits of the air gaps between pole shoes and track $b_{\mathrm{mgk}}(1 \leq \kappa \leq n)$, two branch magnetic circuits of the air gaps between protection blocks and track $b_{\text {pgk }}(1 \leq \kappa \leq 2),(n+1)$ branch magnetic circuits of track $b_{\mathrm{tk}}(1 \leq \kappa \leq n+1)$, and $(n+1)$ loop magnetic circuits $i_{k}(1 \leq \kappa \leq n+1)$. The excitation equation of the second magnetic circuit, which consists of two electromagnets, is expressed as

$$
\begin{aligned}
\frac{1}{2}(\mathrm{IW}+\mathrm{IW})= & \frac{B_{a 2} L_{a 2}}{\mu_{a 2}}+\frac{B_{c 2} L_{c 2}}{\mu_{c 2}}+\frac{B_{\mathrm{mg} 2} L_{\mathrm{mg}}}{\mu_{0}}+\frac{B_{t 2} L_{t 2}}{\mu_{t 2}} \\
& +\frac{B_{\mathrm{mg} 1} L_{\mathrm{mg}}}{\mu_{0}}+\frac{B_{c 1} L_{c 1}}{\mu_{c 1}}
\end{aligned}
$$

where $I$ is the excitation coil current, $A ; W$ is the excitation coil number of turns; $B_{a 2}, B_{c 2}, B_{\mathrm{mg} 2}, B_{t 2}, B_{\mathrm{mg} 1}$, and $B_{c 1}$ is the magnetic induction, $T ; \mu_{a 2}, \mu_{c 2}, \mu_{0}, \mu_{t 2}$, and $\mu_{c 1}$ is the magnetic permeability, $\mathrm{H} / \mathrm{m}$; and $L_{a 2}, L_{c 2}, L_{\mathrm{mg}}, L_{t 2}$, and $L_{c 1}$ is the magnetic path length, $\mathrm{mm}$. As air permeability is much smaller than the permeability of \#10 steel and \#70 Mn steel (Wan, 2013), equation (6) can be simplified as

$$
\mathrm{IW}>\frac{2 B_{\mathrm{mg} 2} L_{\mathrm{mg}}}{\mu_{0}}=\frac{2 \times 0.0019}{1.25 \times 10^{-6}} B_{\mathrm{mg} 2}=3040 B_{\mathrm{mg} 2} \text {. }
$$

According to Figure 2, both \#10 steel and \#70 Mn steel $\mathrm{B}-\mathrm{H}$ curves nearly reach saturation points when $B$ is $1.4 \mathrm{~T}$. Hence, IW should be larger than 4256 ampere-turns. Here, we chose IW as 5000 ampere-turns by assuming the worst scenario.

A coil arrangement form that intertwines three-layer coils on one iron core is proposed. The circuit of three-layer coils is a parallel circuit; therefore, track brake can supply $72 \mathrm{~V}$ as power supply, which is equivalent to the effect of series connection on the iron core. The winding thickness $b_{k}$ is calculated as [11]:

$$
b_{k}=\sqrt[3]{\frac{\rho_{130}(\mathrm{IW} / 3)^{2}}{20 \mu_{m} \cdot f_{k} \cdot \theta \cdot \beta^{2}}}=\sqrt[3]{\frac{0.02339 \times 1675^{2}}{20 \times 0.001289 \times 0.7 \times 90 \times 3^{2}}}=16.49 \mathrm{~mm}
$$

where $\rho_{130}$ is the specific coil coefficient of resistance when the temperature is $130 \mathrm{C}, \Omega \cdot \mathrm{mm}^{2} / \mathrm{m} ; \mu_{m}$ is the coil heat dissipation coefficient, $\mathrm{W} / \mathrm{cm}^{2}{ }^{\circ} \mathrm{C} ; f_{k}$ is the filling coefficient of coil; $\theta$ is the temperature rise of coil, ${ }^{\circ} \mathrm{C}$; and $\beta$ is the depth-width ratio of coil. We ascertain $b_{k}$ as $16 \mathrm{~mm}$. The current density of $j$ is selected as $3.5 \mathrm{~A} / \mathrm{mm}^{2}$ according to the electromagnet design manual. Then, the preliminary sectional area of a single-layer coil $Q_{k}$ is calculated as

$$
Q_{k}=\frac{\mathrm{IW}}{3 \times J \times f_{k}}=\frac{5000}{3 \times 3.5 \times 0.7}=680.3 \mathrm{~mm}^{2} .
$$

The preliminary height of single-layer coil $h_{k}$ is calculated as

$$
h_{k}=\frac{Q_{k}}{b_{k}}=\frac{680.3}{16}=42.5 \mathrm{~mm}
$$

Therefore, the preliminary height of the single-layer coil is determined as $43 \mathrm{~mm}$. The iron core height is ascertained as $160 \mathrm{~mm}$, which has enough space to twine three-layer coils, and the pole shoe height is ascertained as $20 \mathrm{~mm}$.

The gap between adjacent electromagnets $l_{\text {aem }}$ is $5 \mathrm{~mm}$, and the gap between the protection block and the adjacent electromagnet $l_{\text {pem }}$ is $2.5 \mathrm{~mm}$, which serves as a safety gap. To guarantee the functions of protection and cleaning, the length, width, and height of the protection block are set as 100,120 , and $180 \mathrm{~mm}$, respectively. The length, width, and height of the longitudinal beam are 1400,125 , and $75 \mathrm{~mm}$, respectively, which can ensure its functions of protection and installing.

Series-wound coil is concatenated in the primary loop, and it requires few windings. For this reason, the serieswound coil with $H$ skeleton is selected. The thickness of the coil framework is $3 \mathrm{~mm}$. Considering that a considerable amount of heat is generated by rubbing between pole shoes and track, the rank of the coil insulation is selected with an $H$ shape. Moreover, insulating resin is placed between the coil and the iron core for insulation. The thickness of the insulating resin and the electromagnet shell is 2 and $1.5 \mathrm{~mm}$, respectively.

In sum, the adjacent iron core gap $l_{\text {aic }}$ is $50 \mathrm{~mm}$, and the gap between the protection block and the adjacent iron core $l_{\text {pic }}$ is $25 \mathrm{~mm}$.

\section{Simulation and Optimization}

We conduct the optimization of ETB through simulation. First, the theoretical basis of electromagnetic field analysis is illustrated. Then, the assumptions of a finite element model and its simulation results are demonstrated. Finally, optimizations of the number of electromagnets and the pole shoe gap are presented. 


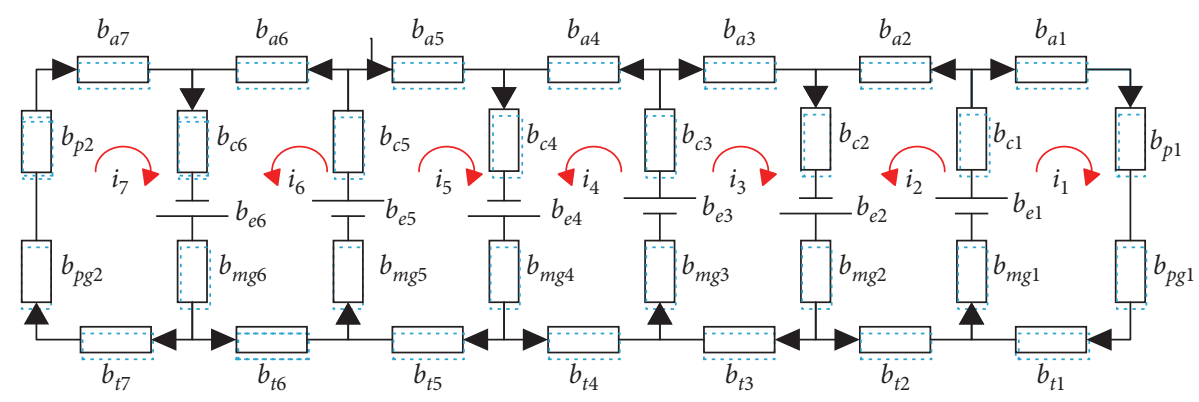

FIGURE 4: Network topology scheme of magnetic circuits.

3.1. Theoretical Basis of Electromagnetic Field Analysis. Numerical analysis of an electromagnetic field involves solving Maxwell equations and other partial differential equations under the given boundary conditions.

The induced electromotive force in a closed loop is proportional to the change rate of magnetic flux through the circuit, which is given as

$$
\oint_{\Gamma} \vec{E} \cdot \mathrm{d} \vec{l}=-\iint_{\Omega} \frac{\partial \vec{B}}{\partial t} \cdot \mathrm{d} \vec{S}
$$

where $E$ is the electric field intensity, $\mathrm{V} / \mathrm{m}$.

The electric flux, which passes through any closed surfaces, equals the electric charge quantity encapsulated by the closed surfaces regardless of the distribution of electrolyte and electric flux density in the electric field. The electric flux can be expressed as

$$
\oiint_{S} \vec{D} \cdot \mathrm{d} \vec{S}=\iiint_{V} \rho_{1} \mathrm{~d} V
$$

where $\rho_{1}$ is the electric charge volume density, $\mathrm{C} / \mathrm{m}^{3}$, and $V$ is the volume area surrounded by closed surfaces.

The line integral of magnetic field intensity along any closed path equals the total current that passes through the hook face, which is determined through this integral path as

$$
\oint_{\Gamma} \vec{H} \cdot \mathrm{d} \vec{l}=\iint_{\Omega}\left(\vec{J}+\frac{\partial \vec{D}}{\partial t}\right) \cdot \mathrm{d} \vec{S}
$$

where $\Gamma$ is the boundary of hook face, $\Omega$; $J$ is the density vector of the conduction current; $\partial D / \partial t$ is the displacement current density, $\mathrm{A} / \mathrm{m}^{2}$; and $D$ is electric flux density, $\mathrm{C} / \mathrm{m}^{2}$.

The magnetic flux that passes through any closed surface equals zero regardless of the distribution of magnetic flux density vector and medium, which is shown as follows:

$$
\oiint_{S} \vec{B} \cdot \mathrm{d} \vec{S}=0
$$

Several physical and engineering parameters are required when solving engineering electromagnetic field problems. Therefore, knowledge of the energy storage of the electric field, the magnetic flux density, the magnetic flux, and calculation of electromagnetic force, and torque is required. The parameters are derived from electric potential and magnetic potential relationships as
$Q=\frac{\varepsilon}{2} \int_{\Omega}|\nabla \phi|^{2} d \Omega=\frac{\varepsilon}{2} \sum_{e=1}^{n} \int_{\Omega^{e}}\left[\left(\frac{\partial \phi}{\partial x}\right)^{2}+\left(\frac{\partial \phi}{\partial y}\right)^{2}+\left(\frac{\partial \phi}{\partial z}\right)^{2}\right] \mathrm{d} \Omega$,

where $Q$ is the energy source term. The energy storage of the electric field can be obtained if the electric field of the electric potential is calculated according to equation (15). The finite element method to achieve total field energy by summing up all unit energies can be expressed as

$$
B=\nabla \times A=x_{\varepsilon} \frac{\partial A}{\partial y}-y_{\varepsilon} \frac{\partial A}{\partial x},
$$

where $A$ is the vector of magnetic potential. Magnetic field power is often expressed by magnetic flux density, $B$, which is calculated by directional derivative of potential function. The magnetic flux can be obtained by the surface integral of the magnetic flux density as

$$
\phi_{m}=\iint_{S} B \mathrm{~d} S=\iint_{S} \nabla \times A \cdot \mathrm{d} S .
$$

Electromagnetic force can be achieved by using virtual displacement after energy storage of electric field through potential function is obtained. Virtual displacement replaces the differential quotient with a difference quotient. Moreover, it calculates magnetic variation to confirm electromagnetic force and electromagnetic torque as follows:

$$
\begin{gathered}
F=\frac{\partial W_{m}}{\partial S}=\frac{\partial W_{m 1}-\partial W_{m 0}}{S_{1}-S_{0}}, \\
T_{m}=P \frac{\partial W_{m}}{\partial \theta}=P \frac{\partial W_{m 1}-\partial W_{m 0}}{\theta_{1}-\theta_{0}},
\end{gathered}
$$

where $W_{m 0}$ is the magnetic energy before virtual displacement; $W_{m 1}$ is the magnetic energy after virtual displacement; $\theta_{0}$ and $S_{0}$ is the location of model before virtual displacement; $\theta_{1}$ and $S_{1}$ is the location of model after virtual displacement.

3.2. Finite Element Model. Finite element simulation of ETB is developed by using a three-dimensional (3D) model to investigate EAF and magnetic field distribution [12]. For the analysis of the present model, the following assumptions are made: 
(1) The process is steady and isothermal

(2) The model is three-dimensional, and it depends on $x$, $y$, and $z$ directions

(3) Adjacent electromagnet current has reverse directions

(4) Waterproof layer, shell, and other structures are ignored because they have little influence on the electromagnetic field [9]

(5) The natural boundary condition and Neumann boundary condition are applied for the model

(6) The ideal air gap between pole shoes and the track is $1 \mathrm{~mm}$

(7) The number of electromagnets is assumed to be six

Ansoft Maxwell is finite element software that specializes in electromagnetic simulation, and it uses electromagnetic field information to predict product performance accurately from physical design information. The software can automatically develop a grid partition. However, some meshes are added manually to increase magnetic field accuracy. According to partition methodology, a refined mesh is carried out in the contact zone to achieve EAF and magnetic distribution precisely. The gridding is composed of fournode tetrahedral finite elements, and the mesh generation parameters are presented in Table 2.

As depicted in Figure 5, a very fine mesh is employed to achieve desired results, which can meet the accuracy of finite element analysis.

3.3. Simulation Results. The convergence results are exhibited in Table 3. The relationships among number of convergence and triangle unit, total energy, and energy error are illustrated intuitively in the table.

As shown in Figure 6, the various colors represent different magnetic field intensities. The color of pole shoes is red, which indicates a large magnetic flux density. The magnetic field gradually strengthens as one move closer to the pole shoe gap because of looping-in that develops between adjacent magnets.

Figure 7 shows the magnetic flux density distribution of a track surface. The six red rectangular blocks correspond to each of the six pole shoes; red, yellow, green, and blue areas primarily exist. The contact areas between pole shoes and track are red, which indicates strong magnetic flux density. The magnetic flux density of other areas gradually weakens the farther one is from pole shoes. Figure 8 shows the magnetic flux density distribution of the bottom of pole shoes, whose distribution rules are similar to those showed in Figure 7.

We obtain different EAFs by setting different current excitations. As shown in Table 4, the EAF is greater when ampere-turn is added. However, the EAF increases very little when the current excitation is more than 5000 ampere-turns, which means that the force has nearly reached the limit. Hence, the choice of 5000 ampere-turns as the current excitation is a reasonable decision.
TABLE 2: Mesh generation parameters.

\begin{tabular}{lcc}
\hline Component & $\begin{array}{c}\text { Maximum element length } \\
(\mathrm{mm})\end{array}$ & $\begin{array}{c}\text { Element } \\
\text { number }\end{array}$ \\
\hline Iron core & 1 & 60,000 \\
Pole shoe & 1 & 40,000 \\
Track & 1 & 65,000 \\
Block & 1 & 11,400 \\
Coil & 2 & 21,000 \\
Longitudinal & 2 & 32,000 \\
beam & & \\
\hline
\end{tabular}

3.4. Optimization of ETB. The electromagnet number and the pole shoe gap are optimized in this part to improve the EAF between ETB and track by using Ansoft Maxwell.

Different EAFs are achieved with the variation of magnet number when the effective air gap between pole shoes and track is $1 \mathrm{~mm}$. The current excitation of coil varies at 1630 , 3300, and $5000 \mathrm{~A}$, respectively. As shown in Table 5, EAF is at its maximum when the magnet number is six. However, the magnetic circuit number will decrease as the electromagnet length increases. Moreover, decreased electromagnet length will lead to a serious decline in single magnetic flux $[5,13]$.

The gap between adjacent pole shoes has a certain influence on EAF. The leakage flux will increase if the gap is too small. However, the working area between pole shoes and track will reduce when the gap is too large, which will decrease the main flux $[5,13]$. Thus, the gap has an optimum value when the electromagnet is determined.

Table 6 shows the relationship between the pole shoe gap and EAF when a $10 \mathrm{~mm}$ step change of the pole shoe gap is used. EAF reduces when the pole shoe gap is more than $40 \mathrm{~mm}$ due to the decrease in the effective friction area between pole shoes and track. EAF reduces when the pole shoe gap is less than $40 \mathrm{~mm}$ because the leakage flux between adjacent pole shoes increases. Therefore, the gap between adjacent pole shoes is determined as $40 \mathrm{~mm}$.

\section{Final Design of ETB Based on the Optimizations}

The final design of ETB based on the optimization results is obtained in this section. The iron core length is determined by ascertaining six electromagnets. The copper wire diameter, coil turns, and the height of the single-layer coil are calculated based on iron core length. Then, the integrated parameters of ETB are presented.

4.1. Design of Magnetic Circuit Coil Based on Optimizations. The iron core length $D_{1}$ is $150 \mathrm{~mm}$ when the magnet number is six, which satisfies the constraint equation (2). Then, we can confirm the average diameter of coil as

$$
D_{\mathrm{cp}}=\frac{D_{1}+f}{2}+b_{k}=\frac{150+75}{2}+16=128.5 \mathrm{~mm}
$$




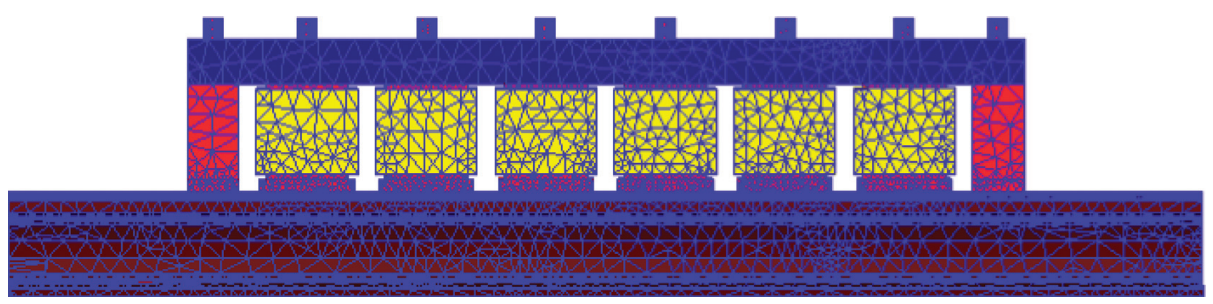

FIGURE 5: Grid partition model.

TABle 3: Convergence data.

\begin{tabular}{lcccc}
\hline Pass & \# Tetrahedra & Total energy (\%) & Energy error (\%) & Delta energy (\%) \\
\hline 1 & 273,788 & 509.81 & 23.168 & N/A \\
2 & 355,874 & 478.66 & 5.0929 & 0.91799 \\
3 & 462,674 & 474.27 & 3.0581 & 0.32446 \\
4 & 601,462 & 470.41 & 1.2327 & 0.06139 \\
\hline
\end{tabular}

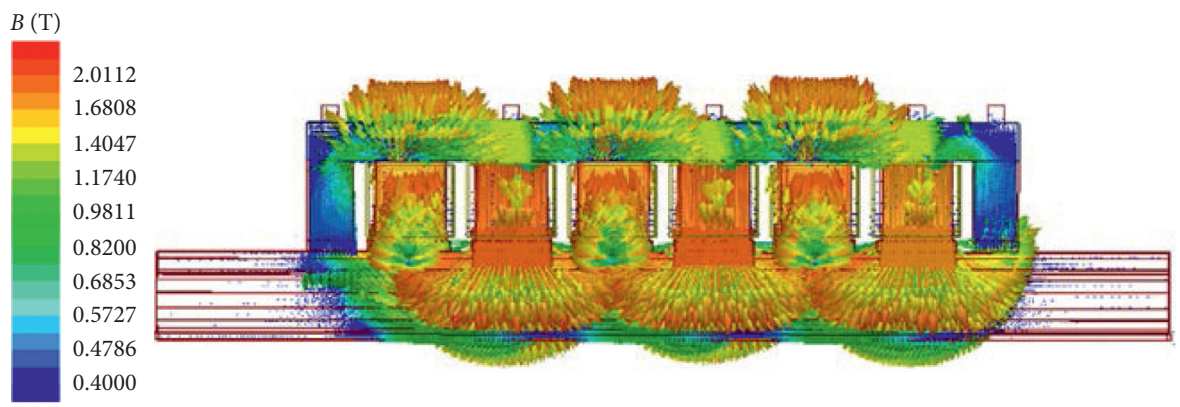

FIgURE 6: Vector magnetic flux density.

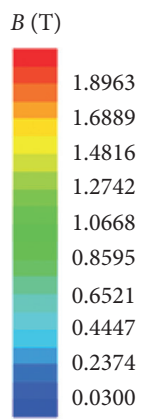

Figure 7: Magnetic flux density on the track surface.

B (T)
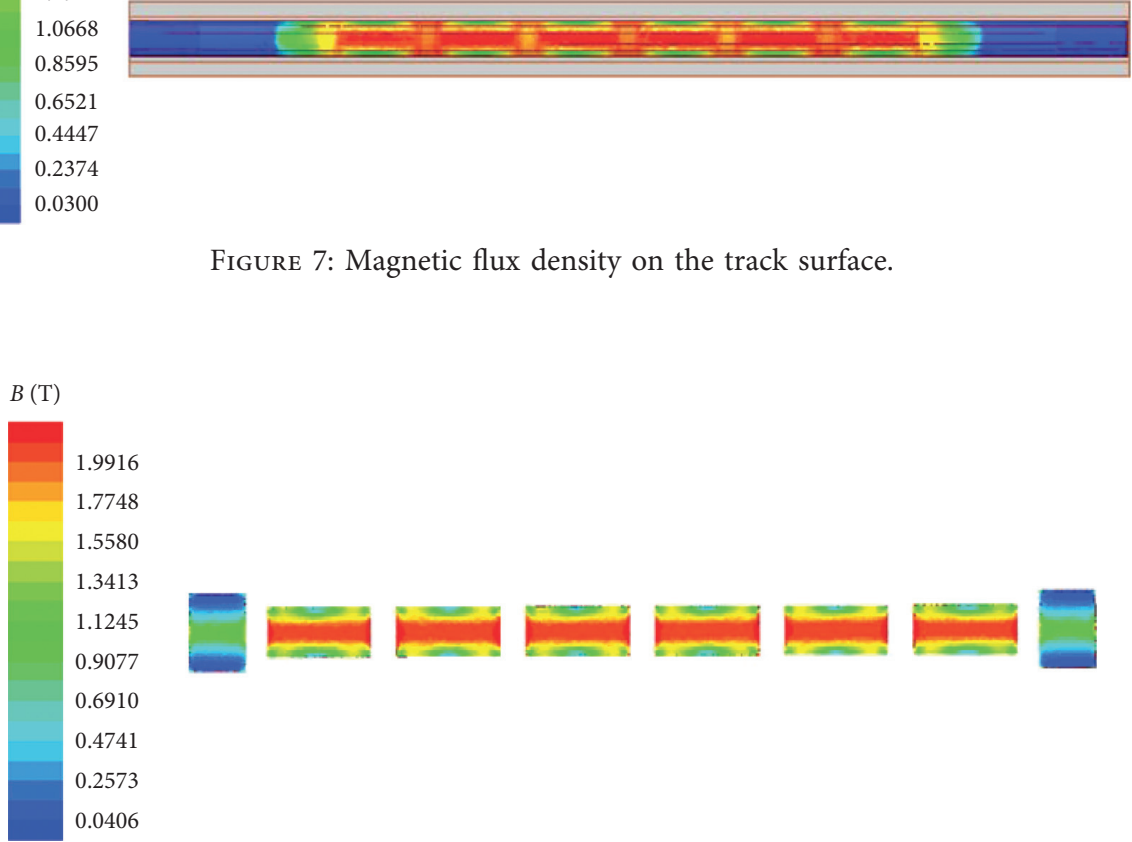

2374

0300
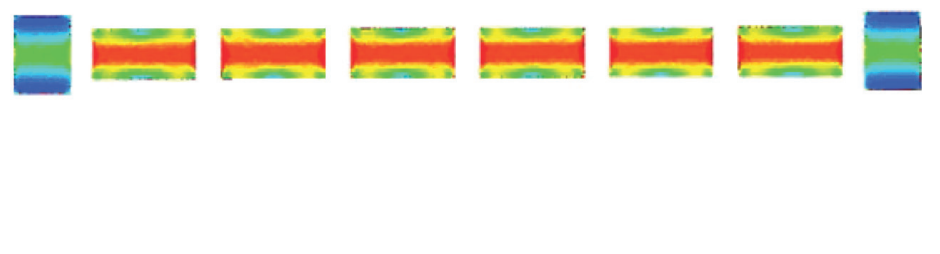

Figure 8: Magnetic flux density on the pole shoes surface. 
TABLE 4: Relationship between ampere-turns and attractive force.

\begin{tabular}{lcccc}
\hline Coil excitation (ampere-turn) & 1630 & 3300 & 5000 & 6000 \\
\hline Attractive force $(\mathrm{N})$ & 39,722 & 50,181 & 61,401 & 62,397 \\
\hline
\end{tabular}

TABLE 5: Attractive force at different electromagnet number and current excitation.

\begin{tabular}{|c|c|c|c|c|c|}
\hline \multirow{2}{*}{ Current excitation (ampere-turn) } & \multicolumn{5}{|c|}{ Attractive force $(\mathrm{N})$} \\
\hline & Four magnets & Five magnets & Six magnets & Seven magnets & Eight magnets \\
\hline 1630 & 20,899 & 28,404 & 39,722 & 37,522 & 32,350 \\
\hline 3300 & 26,402 & 35,883 & 50,181 & 47,401 & 40,860 \\
\hline 5000 & 32,305 & 43,906 & 61,401 & 58,032 & 50,152 \\
\hline 6000 & 34,419 & 45,465 & 62,397 & 58,904 & 50,442 \\
\hline
\end{tabular}

TABLE 6: Relationship between pole shoe gap and attractive force.

\begin{tabular}{lccccc}
\hline Pole shoe gap $(\mathrm{mm})$ & 50 & 40 & 30 & 20 & 10 \\
\hline Attractive force $(\mathrm{N})$ & 60,728 & 61,401 & 60,751 & 58,116 & 56,542 \\
\hline
\end{tabular}

As magnetic potential is generated when the current passes through the coil winding, the equations of voltage and magnetic potential are expressed as follows (Wan, 2013):

$$
\left.\begin{array}{l}
q=\frac{\pi}{4} d^{2} \\
R=\frac{4 \rho D_{\mathrm{cp}} W}{q} \\
U=\mathrm{IR}=\frac{\mathrm{IW}}{W} R=\frac{\mathrm{IW}}{W} \frac{4 \rho D_{\mathrm{cp}}}{q} W=\frac{16 \rho D_{\mathrm{cp}} \mathrm{IW}}{\pi d^{2}}
\end{array}\right\},
$$

where $U$ is the supply voltage, $\mathrm{V} ; R$ is the field coil resistance, $\Omega ; \rho$ is the coil coefficient of resistance, $\Omega \cdot \mathrm{mm}^{2} / \mathrm{m} ; D_{\text {cp }}$ is the mean diameter of excitation coil, $m$; and $q$ is the crosssectional area of bare copper wire, $\mathrm{mm}^{2}$. The copper wire diameter can be calculated according to equation (20) as

$d=\sqrt{\frac{16 \rho_{130} D_{\mathrm{cp}} \mathrm{IW}}{\pi U}}=\sqrt{\frac{16 \times 0.02339 \times 0.1285 \times 1675}{3.14 \times 24}}=1.0339 \mathrm{~mm}$.

The copper wire diameter $d$ is determined as $1.04 \mathrm{~mm}$, and the outer diameter of enamelled wire $d_{1}$ is chosen as $1.12 \mathrm{~mm}$ according to the enameled wire coil specification table [11]. Electromagnet coil calorific value is an important factor in electromagnet design. Furthermore, the maximum temperature of coil must be under the allowable value [14].

$$
\left.\begin{array}{l}
\theta=\frac{p}{\mu_{m} S}=\frac{U^{2}}{R \mu_{m} S} \\
S=S_{H}+\eta_{m} S_{\theta}
\end{array}\right\},
$$

where $p$ is the coil power, $W$; $S$ is the heat dissipating area, $\mathrm{mm}^{2} ; S_{H}$ is the outer surface area of the coil, $\mathrm{mm}^{2} ; S_{\theta}$ is the inner surface area of the coil, $\mathrm{mm}^{2}$; and $\eta_{m}$ is the coil factor, $\mathrm{mm}^{2}$. The heat radiating area $S$ and the coil resistance $R$ can be calculated according to equation (22).

$$
\begin{aligned}
S= & S_{H}+\eta_{m} S_{\theta}=(75+150+16 \times 4) \times 2 \times 45+2 \\
& \times(75+150) \times 2 \times 45=66510 \mathrm{~mm}^{2}, \\
R \geq & \frac{U^{2}}{\mu_{m} S \theta}=\frac{24 \times 24}{0.001204 \times 665.1 \times 90}=7.99 \Omega .
\end{aligned}
$$

$R$ is ascertained as $8.5 \Omega$. Moreover, $I$ and $W$ are confirmed as follows:

$$
\begin{gathered}
I=\frac{U}{R}=\frac{24}{8.5}=2.8 \mathrm{~A} . \\
W=\frac{I W}{I}=\frac{1675}{2.8}=600 \text { Turns. }
\end{gathered}
$$

The calorific value of the coil should be limited to ensure that the current density value meets certain requirements. Therefore, the current density value of the coil is obtained as

$$
j=\frac{I}{q}=\frac{4 \times 2.8}{3.14 \times 1.04^{2}}=3.3 \mathrm{~A} / \mathrm{mm}^{2} .
$$

The current density value satisfies the electromagnet design requirement for uninterrupted operation [14]. The filling coefficient of the coil, the coil sectional area, and the coil height are calculated as follows:

$$
\begin{aligned}
f_{k} & =\frac{\pi}{4}\left(\frac{d}{d_{1}}\right)=\frac{3.14}{4} \times\left(\frac{1.04}{1.12}\right)^{2}=0.68, \\
Q_{k} & =\frac{q \times W}{f_{k}}=\frac{0.849 \times 600}{0.68}=749.1 \mathrm{~mm}^{2}, \\
h_{k} & =\frac{Q_{k}}{b_{k}}=\frac{749.1}{16}=46.8 \mathrm{~mm} .
\end{aligned}
$$

Therefore, the final height of the single-layer coil is designed as $47 \mathrm{~mm}$. The three-layer coils of one electromagnet can supply 5040 ampere-turns according to 
TABLE 7: Parameters of the ETB.

\begin{tabular}{lcc}
\hline Component & Parameter & Data \\
\hline Iron core & Length $\times$ width $\times$ eight & $150 \mathrm{~mm} \times 75 \mathrm{~mm} \times 160 \mathrm{~mm}$ \\
\hline Pole shoe & Length $\times$ width $\times$ height & $160 \mathrm{~mm} \times 75 \mathrm{~mm} \times 20 \mathrm{~mm}$ \\
& Gap & $40 \mathrm{~mm}$ \\
& Inner diameter & $160 \mathrm{~mm} \times 85 \mathrm{~mm}$ \\
& Coil resistance & $130^{\circ} \mathrm{C} 8.5 \Omega$ \\
& Room temperature & $8 \Omega$ \\
Coil & Height & $47 \mathrm{~mm} \times 3$ \\
& Thickness & $16 \mathrm{~mm}$ \\
& Working voltage & $24 \mathrm{~V}$ \\
& Magnetic potential & $1675 \times 3 \mathrm{ampere}$-turns \\
Electromagnet & Outer diameter & $195 \mathrm{~mm} \times 120 \mathrm{~mm}$ \\
& Number & 6 \\
Protection block & Gap & $5 \mathrm{~mm}$ \\
Longitudinal beam & Length $\times$ width $\times$ height & $100 \mathrm{~mm} \times 120 \mathrm{~mm} \times 180 \mathrm{~mm}$ \\
& Gap & $2.5 \mathrm{~mm}$ \\
\end{tabular}

equations (24) and (25), which can satisfy the requirement of 5000 ampere-turns.

4.2. Integrated Design of ETB. Considering the abovementioned relations, the thickness of the coil, framework, insulating resin, and the electromagnet shell is $16,3,2$, and $1.5 \mathrm{~mm}$, respectively. Hence, the width $w_{\mathrm{em}}$ and length $l_{\mathrm{em}}$ of the electromagnet is calculated as follows:

$$
\begin{gathered}
w_{\mathrm{em}}=75+16 \times 2+3 \times 2+2 \times 2+1.5 \times 2=120 \mathrm{~mm}, \\
l_{\mathrm{em}}=150+16 \times 2+3 \times 2+2 \times 2+1.5 \times 2=195 \mathrm{~mm} .
\end{gathered}
$$

The parameters of the ETB are illustrated in Table 7.

\section{Experimental Verification of EAF}

We develop a prototype based on optimized parameters. Then, static tests of EAF and a comparison between tests and simulations are carried out. Finally, the electromagnetic BF (EBF) of ETB is calculated in the next section.

5.1. Prototype Manufacture. We develop a 3D model by using CATIA software according to the parameters in Table 7. Figure 9(a) indicates the electromagnet structure, and Figure 9(b) represents the assembly drawing of ETB.

5.2. Attractive Force Test. Figure 10(a) shows a picture of the final prototype assembly. The threaded holes on the top of the longitudinal beam are used to install rings when performing the experiment. The holes on the side face of the longitudinal beam are used to install a suspension system of ETB. Figure 10(b) shows the assembly, where the ETB is connected to an electronic scale through rings and a pendant chain and is lifted up gradually by a traveling crane. Track is fixed on the floor by U-profile steels. The numerical value of EAF and track brake weight can be directly read from an electronic scale when track brake is separated from track.
The numerical value of track brake weight is a constant value. Therefore, the EAF is easy to obtain.

5.3. Contrastive Analyses of Tests and Simulations. The average value of EAF is achieved as the final result through many experiments. The contrastive analyses of tests and simulations are exhibited in Table 8 .

The EAF of tests agrees with that of the simulations. The error is within the acceptable range. The following explanations for discrepancies between the test and the simulation are given after careful observations of the test process:

(1) Negative factors, such as pits and dust on track surface, have some effect on the experiment result. In addition, the contact between pole shoes and track may be incomplete due to assembly error.

(2) The material property of ETB is constant during the simulation, while temperature influences copper wire resistance during the test.

(3) The magnetizing curve used in the simulation is referred to as the electrical engineering handbook, whose value is slightly larger than the actual value [11].

\section{Estimation of EBF}

A facility for a dynamic EBF test is unavailable. Therefore, $\mathrm{EBF}$ and braking deceleration are estimated by using friction coefficient [15].

As the weight of $\mathrm{CRH}_{2} M$ is $441,180 \mathrm{~kg}$, its air braking force $F_{\mathrm{ab}}$ is expressed as [14]

$$
F_{\mathrm{ab}}=510170 \times \frac{v+150}{2 v+150}
$$

where $v$ is train speed, $\mathrm{km} / \mathrm{h}$. $\mathrm{CRH}_{2}$ has eight carriages, and it can be equipped with 16 ETBs [16]. According to the empirical equation from a Russian Federation laboratory, the friction coefficient of track $\mu_{k}$ and the EBF $F_{\mathrm{eb}}$ are expressed as [17] 


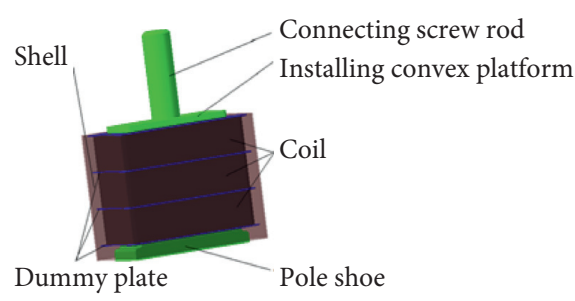

(a)

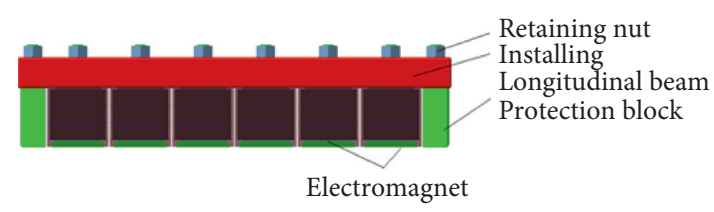

(b)

FIgURE 9: (a) Electromagnet structure and (b) assembly drawing of ETB.
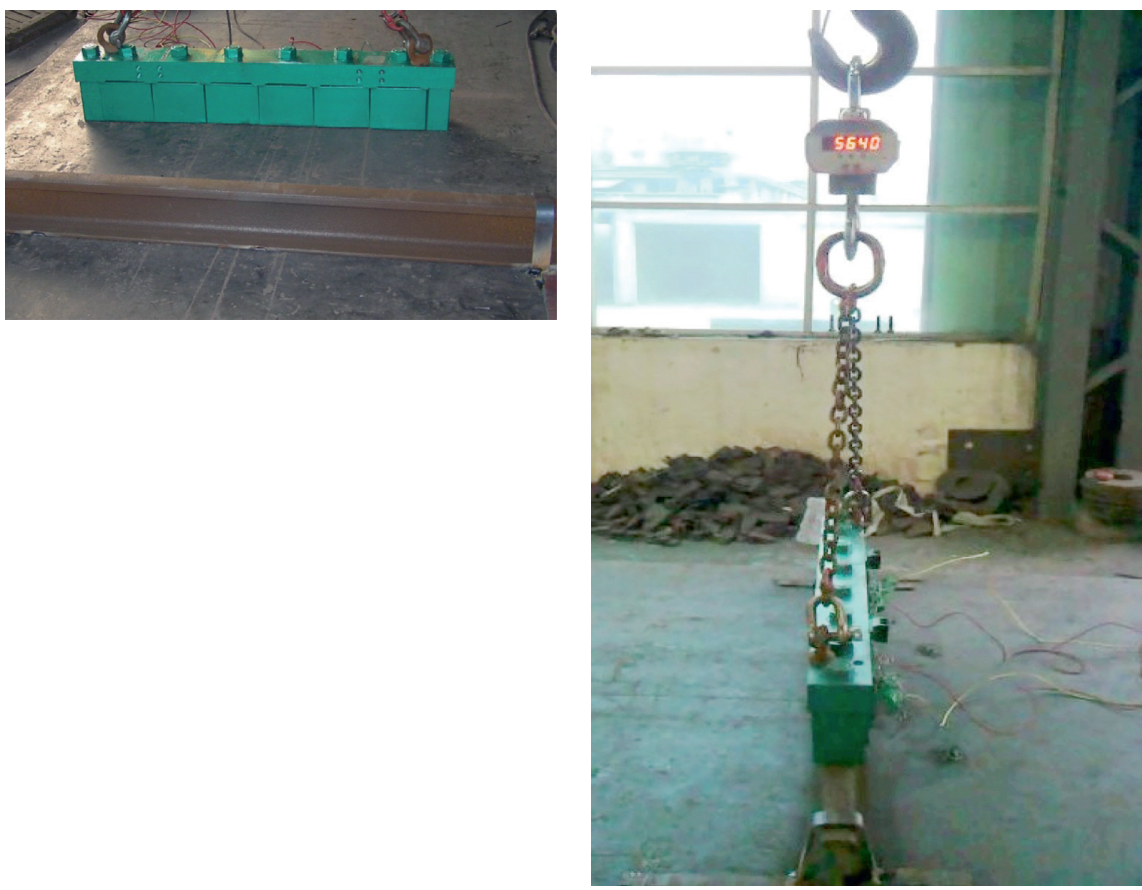

(a)

(b)

Figure 10: (a) Assembly drawing of prototype. (b) Attractive force test.

TABLE 8: Comparison between the results of test and simulation.

\begin{tabular}{lccc}
\hline Magnetic potential (ampere-turn) & Test force $(\mathrm{N})$ & Simulation force $(\mathrm{N})$ & Error $(\%)$ \\
\hline 5000 & 56,940 & 61,401 & 7.83 \\
3300 & 48,000 & 50,181 & 4.54 \\
1630 & 36,120 & 39,722 & 9.97 \\
\hline
\end{tabular}

$$
\begin{aligned}
\mu_{k} & =0.19 \times \frac{10.8 v+100}{21.6 v+100} . \\
F_{\mathrm{eb}} & =56940 \times 16 \times \mu_{k}=173097.6 \times \frac{10.8 v+100}{21.6 v+100} .
\end{aligned}
$$

As the running resistance of $\mathrm{CRH}_{2}$ is too small compared with the air $\mathrm{BF}$ and the $\mathrm{EBF}$, the braking deceleration of $\mathrm{CRH}_{2} a_{\mathrm{b}}$ is calculated according to equations (30) and (32) as

$$
a_{b}=\frac{F_{\mathrm{ab}}}{M}+\frac{F_{\mathrm{eb}}}{M}=1.16 \times \frac{v+150}{2 v+150}+0.39 \times \frac{10.8 v+100}{21.6 v+100} .
$$

The single ETB installed in the German ICE can provide $41,237 \mathrm{~N} \cdot \mathrm{EAF}$. When it is used for $\mathrm{CRH}_{2}$, the electromagnetic braking deceleration of $\mathrm{CRH}_{2} a_{\mathrm{Ib}}$ is expressed as

$$
a_{\mathrm{Ib}}=1.16 \times \frac{v+150}{2 v+150}+0.28 \times \frac{10.8 v+100}{21.6 v+100} .
$$




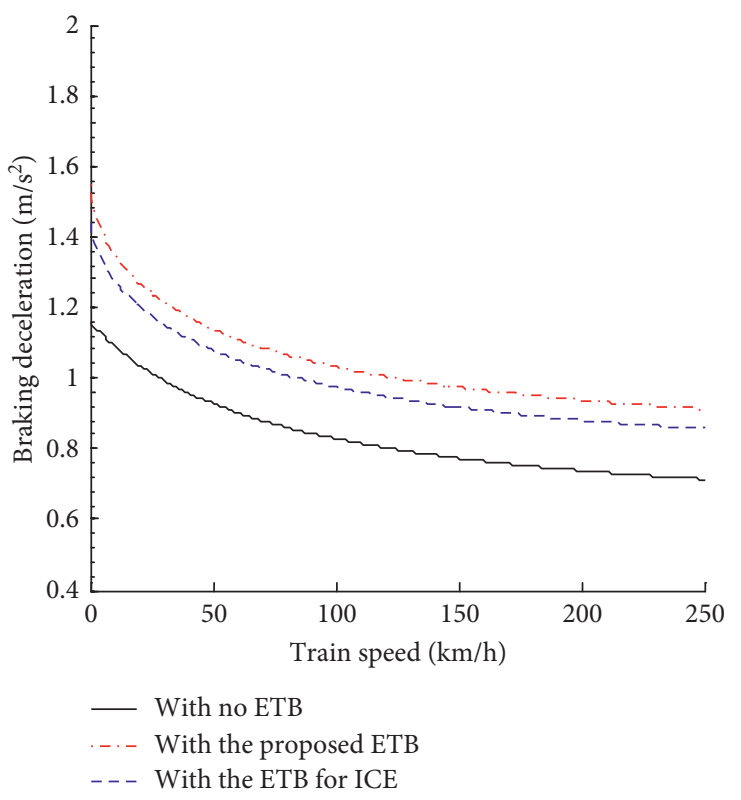

FigURE 11: Emergency brake deceleration curves.

As shown in Figure 11, the proposed ETB can provide $0.20 \mathrm{~m} / \mathrm{s}^{2}$ braking deceleration, while ETB for ICE can provide $0.14 \mathrm{~m} / \mathrm{s}^{2}$ braking deceleration when the train speed is $200 \mathrm{~km} / \mathrm{h}$. Therefore, the proposed ETB can enhance braking deceleration by $39 \%$ because of its longitudinal magnetic circuit and its functioning.

\section{Conclusions}

In the present study, a new ETB is proposed that has the following two outstanding characteristics: first, longitudinalaxis magnetic circuits excited by electromagnets with multiple coils, and second, a design in which pole shoes extend outwards. The proposed ETB is optimized to improve the BF, the electromagnet number is optimized to be six, and the pole shoe gap is optimized to be $40 \mathrm{~mm}$. Moreover, experimental verification of electromagnetic attractive force is performed through prototype tests, and the newly developed electromagnetic track brake can enhance electromagnetic braking deceleration by $39 \%$.

\section{Data Availability}

The calculated data used to support the findings of this study are included within the article.

\section{Conflicts of Interest}

The authors declare that there are no conflicts of interest regarding the publication of this paper.

\section{Acknowledgments}

This work was supported by the National Natural Science Foundation of China (Grant no. 51575239).

\section{References}

[1] C. Fan, F. Dou, B. Tong et al., "Risk analysis based on ahp and fuzzy comprehensive evaluation for maglev train bogie," Mathematical Problems in Engineering, vol. 2016, Article ID 1718257, 10 pages, 2016.

[2] K. Gao, Z. Huang, J. Peng et al., "An online distributed game optimal control for heavy haul trains with limited communication," Mathematical Problems in Engineering, vol. 2015, Article ID 158695, 9 pages, 2015.

[3] H. David, "All aboard for high-speed rail," Mechanical Engineering, vol. 118, pp. 94-97, 1996.

[4] M. J. Leigh, "Brake blending," Proceedings of the Institution of Mechanical Engineers, Part F: Journal of Rail and Rapid Transit, vol. 208, no. 1, pp. 43-49, 1994.

[5] O. Arias-Cuevas and Z. Li, "Field Investigations into the performance of magnetic track brakes of an electrical multiple unit against slippery tracks. part 2: braking force and side effects," Proceedings of the Institution of Mechanical Engineers, Part F: Journal of Rail and Rapid Transit, vol. 226, no. 1, pp. 72-94, 2012.

[6] L. Zhang and X. Zhuan, "Braking-penalized receding horizon control of heavy-haul trains," IEEE Transactions on Intelligent Transportation Systems, vol. 14, no. 4, pp. 1620-1628, 2013.

[7] M. Yao and L. Wang, "Analysis and simulation on the influencing factors of braking force for permanent magnetic brake," Applied Mechanics and Materials, vol. 278-280, pp. 278-281, 2013.

[8] A. R. Albrecht, P. G. Howlett, P. J. Pudney, and X. Vu, "Energy-efficient train control: from local convexity to global optimization and uniqueness," Automatica, vol. 49, no. 10, pp. 3072-3078, 2013.

[9] M. Jou, J. K. Shiau, and C. C. Sun, "Design of a magnetic braking system," Journal of Magnetism and Magnetic Materials, vol. 304, no. 1, pp. e234-e236, 2006.

[10] G. Gfatter, S. Haas, and G. Vohla, Track Brakes Handbook, Knorr-Bremse AG, Munich, Germany, 2004.

[11] Y. Wan, Electrical Engineering Handbook, China Electric Power Press, Beijing, China, 2013.

[12] L. Zhang and X. Zhuan, "Optimal operation of heavy-haul trains equipped with electronically controlled pneumatic brake systems using model predictive control methodology," IEEE Transactions on Control Systems Technology, vol. 22, no. 1, pp. 13-22, 2014.

[13] S. L. Lu, Y. F. Gu, S. A. Chen, and S. Wang, "A numerical method of the electromagnetic force for the electromagnetic track brake," Applied Mechanics and Materials, vol. 253-255, pp. 2160-2162, 2013.

[14] S. Wang, Probe into Wear Behavior of EMB's Pole Shoe and Rail, Unpublished Master's Thesis, Jiangsu University, Zhenjiang, China, 2014.

[15] C. Braccesi, F. Cianetti, and R. Scaletta, "Development of a new procedure for the wheel-rail contact force evaluation in simulations of railway dynamics," International Journal of Heavy Vehicle Systems, vol. 12, no. 2, pp. 69-86, 2005.

[16] X. Peng, Y. Y. Zhou, Q. Y. Li, and X. Q. He, "Brake distance calculation of CRH," Applied Mechanics and Materials, vol. 380-384, pp. 201-204, 2013.

[17] M. Dhanasekar, C. Cole, and Y. Handoko, "Experimental evaluation of the effect of braking torque on bogie dynamics," International Journal of Heavy Vehicle Systems, vol. 14, no. 3, pp. 308-330, 2007. 